\title{
Norma internacional International norm
}

\author{
Ruth Martinón Quintero \\ Universidad de La Laguna \\ ORCID ID 0000-0001-8013-7215 \\ ruth.martinon@ull.es
}

\section{Cita recomendada:}

Martinón Quintero, R. (2020). Norma internacional. Eunomía. Revista en Cultura de la Legalidad, 18, pp. 274-284.

doi: https://doi.org/10.20318/eunomia.2020.5277

\section{Resumen}

Recibido / received: $12 / 02 / 2020$

Aceptado / accepted: 04/03/2020

La norma internacional es especialmente adaptativa a la realidad de la sociedad internacional que regula. Los cambios en la estructura del ordenamiento jurídico internacional a través de su institucionalización y humanización, por un lado, y el mismo proceso de globalización, por otro, han redefinido la caracterización de la norma internacional. No solo se trata de la expansión de la materia objeto de regulación y el incremento de los actores que participan más o menos formalmente en su formación, sino también en la misma caracterización de la norma internacional como elemento del ordenamiento jurídico, al dotarse éste de jerarquía y, pese a su ampliación y complicación, mantener la sistematicidad.

\section{Palabras clave}

Tratado, costumbre, jurisprudencia, derecho blando, ius cogens, erga omnes, formación del derecho internacional, Estado, organización internacional, actores internacionales, sociedad internacional

\begin{abstract}
The international norm is especially adaptive to the reality of the international society it regulates. Changes in the structure of the international legal system through its institutionalization and humanization, on the one hand, and the process of globalization, on the other, have redefined the characterization of the international norm. It is not only about the expansion of the subject matter of regulation and the increase of the actors that participate more or less formally in its formation, but also in the same characterization of the international norm as an element of the legal system, when it is endowed with hierarchy and, despite its expansion and complication, maintains systematicity.
\end{abstract}

\section{Keywords}

Treaty, costumary law, soft law, ius cogens, erga omnes, formation of International Law, State, international organization, international actors, international society. 
SUMARIO. 1. Introducción. 2. La relevancia del elemento histórico. 2.1. La globalización. 2.2. La institucionalización y humanización del derecho internacional. 3. El derecho internacional público como sistema. 4.Reflexiones finales.

\section{Introducción}

Entendemos por normas internacionales las reglas jurídicas que junto a los principios forman el ordenamiento jurídico de la Sociedad internacional contemporánea (Díez de Velasco, 2017, p. 75). El interés que puede tener una reflexión sobre estas normas internacionales parte de que el ordenamiento jurídico internacional tiene una caracterización propia y marcadamente distinta de la de los ordenamientos jurídicos internos de los Estados. Una de las razones es la profunda variación que experimenta en función de los cambios protagonizados por la sociedad que regula, la sociedad internacional. Aunque la interdependencia entre norma jurídica y sociedad regulada por ella es propia de todos los ordenamientos jurídicos (sic societas, sicut ius), es aún más evidente en el ordenamiento jurídico internacional en la medida en que estos cambios no solo se aprecian en el contenido material de las regulaciones jurídicas, sino en la misma naturaleza y características de las fuentes formales del derecho internacional $\mathrm{e}$, incluso, en quiénes pueden ostentar la subjetividad jurídico internacional.

Por todo ello es relevante detenerse en el marco histórico de la norma internacional. A continuación, atenderemos a su incardinación en el ordenamiento jurídico internacional como sistema, en la medida en que consideramos que la reflexión sobre la sistematicidad o no de este ordenamiento es también un aspecto importante para la comprensión de la norma internacional en nuestros días.

\section{La relevancia del marco histórico}

La referencia al momento histórico es un factor imprescindible para acercarnos a una comprensión de la norma internacional que vaya más allá de la conceptualización formal que la identifica simplemente con la norma creada por los procedimientos propios del derecho internacional. De hecho, atender a esos procedimientos ya nos obliga a abordar al referente histórico.

En concreto, el proceso de adaptación del derecho internacional de nuestros días es fruto de dos procesos interrelacionados pero distintos: el fenómeno de la globalización, y la institucionalización y correlativa humanización del derecho internacional. Ambos afectan a la tradicional omnipotencia del Estado en la configuración de este ordenamiento jurídico y ello tiene consecuencias directas en la caracterización de la norma internacional.

\subsubsection{La globalización}

La globalización es el fenómeno que no solo supone la unificación del espacio y el tiempo terrestres, con la consiguiente intensificación de las interacciones internacionales, sino que, como explica el profesor Celestino del Arenal (2008, p. 216), conlleva también la instantaneidad de dichas relaciones y el incremento de la conciencia de los seres humanos de pertenecer a un mundo único. Este fenómeno no es solo económico, la política se deslimita y se desestataliza (Del Arenal, 2008, p. 217 ), la mayor parte de los retos son globales, surgen nuevos actores en la sociedad 
internacional y, todo ello, supone límites fácticos a la soberanía del Estado en su concepción tradicional (Palomares, 2006, p. 26).

Este fenómeno tiene diferentes efectos sobre el ordenamiento jurídico internacional. En primer lugar, la profunda interdependencia de los actores, los espacios y los acontecimientos, supone una demanda creciente de la intervención del derecho internacional (Boyle y Chinquin, 2007, p. 19). El terrorismo, el deterioro del medio ambiente y el cambio climático, los movimientos migratorios, los movimientos de mercancías y capitales, el tráfico de armas y estupefacientes, entre otros muchos desafíos, requieren la cooperación en términos jurídicos más allá de las fronteras nacionales. De este modo, la ampliación de los ámbitos regulados por el derecho internacional público ha hecho que sea difícil encontrar un espacio material que quede reservado al ámbito intraestatal.

Si antaño un elemento definitorio del derecho internacional era el ámbito de relaciones que regulaba, actualmente este aspecto sustancial o material no puede cumplir esa función definitoria. De hecho, ya Kelsen (2013/1952, pp. 156-157) había observado que el ámbito de regulación de este ordenamiento jurídico es potencialmente ilimitado. Sin embargo, esta ampliación del campo objeto de regulación sí afecta a la modelación de la norma internacional desde dos perspectivas básicas. Por un lado, porque aunque este proceso de globalización ha impulsado el aumento de las regulaciones internacionales en general, se puede apreciar que dicho fenómeno ha supuesto, a su vez, una especial proliferación del tratado como tipo de norma internacional idónea para regular cuestiones técnicas que requieren regulación en detalle. También se genera el incremento del número de sistemas de resolución de controversias, incluyendo la creación de nuevos tribunales internacionales. Precisamente, es la necesidad común de hacer frente a los fenómenos que no respetan las fronteras estatales lo que obliga a los Estados a trascender las relaciones de coexistencia para abordar las de cooperación (Carrillo Salcedo, 1997, p. 588). Las normas para la cooperación expresan un haz de valores e intereses comunes mucho más amplio que en las normas meramente reguladoras de coexistencia.

En segundo lugar, si como veremos, el proceso de institucionalización y humanización del Derecho interncaional acarrea el planteamiento de cuestiones difíciles sobre la subjetividad internacional, el proceso de globalización permite que nuevos actores, distintos del Estado, tengan capacidad para participar en el escenario internacional, incluidos los procesos de creación de las normas, con independencia de su consideración o no como sujetos de derecho internacional. Ello acontece, asimismo, en un escenario en el que también se desdibuja la frontera entre público y privado, lo que en la doctrina anglosajona Koh ha sintetizado en el concepto de derecho trasnacional (1996, p. 183). Con él describe un proceso en el que múltiples actores públicos y privados, nacionales e internacionales construyen, interpretan, internalizan y ejecutan las normas de ese derecho trasnacional que, entre otras cosas, sirve para explicar por qué actores poderosos como Estados Unidos acaban cumpliendo preceptos del derecho internacional que les resultan incómodos. El tiempo nos dirá si estamos ante una combinación de derecho interno y derecho internacional, o ante un Derecho conceptualmente distinto sobre el que incluso se puede dudar que sea Derecho (Cotterrell, 2012, pp. 501-504).

Ciertamente, las organizaciones internacionales, pero también los tribunales internacionales y nacionales, las empresas trasnacionales, los actores públicos subestatales, las organizaciones no gubernamentales y los seres humanos pueden afectar, y con frecuencia lo hacen, el proceso de creación normativa, aunque los Estados mantengan la última palabra en la medida en que el carácter relacional del derecho internacional se muestra irreductible (Carrillo Salcedo, 1997, p. 595). 
No solo se ha otorgado a muchas ONGs un papel consultivo en órganos de Naciones Unidas, y son conocidos los casos en los que han logrado el desarrollo de esferas tan importantes del derecho internacional como el derecho humanitario (impulsado por el Comité Internacional de la Cruz Roja), la influencia en la determinación del denominado derecho consuetudinario trasnacional (Müller, 2008, p. 24) y la firma de importantes tratados internacionales (como la Convención contra la tortura , impulsado por Amnistía Internacional; o la Convención contra la minas antipersona, de nuevo impulsada por una campaña del Comité Internacional de la Cruz Roja; entre otros). Incluso las personas individualmente consideras han tenido grandes logros como el del profesor Rapahel Lemkin que definió el genocidio y logró su penalización internacional.

Esta realidad ha hecho que se haya llegado a plantear que las actividades de las ONGs sean aceptadas como prácticas que pueden constituir normas internacionales consuetudinarias, como recogen Boyle y Chinkin (2007, p. 36). Sin embargo, es importante diferenciar el papel de grupo de presión que, en su caso, puede regularse como tal, por ejemplo de las ONGs, y el reconocimiento de la condición de sujeto de derecho internacional. En el caso de las personas, esa personalidad jurídico internacional parecer estar conformándose progresivamente; pero no por ese posible papel de influencia en la creación normativa, sino por su condición de titulares de los derechos humanos, la responsabilidad penal internacional, y la posibilidad de ius estandi activo y pasivo al efecto. De modo que, de acabar consolidándose este estatus para las personas, existiría una subjetividad jurídico internacional que no acarrearía la capacidad de crear derecho (como sí sucede con los Estados o las organizaciones internacionales).

También es evidente el papel de las empresas trasnacionales a través de los tratados de inversión que amparan sus intervenciones en Estados distintos al de la matriz, y los contratos que firman con gobiernos colocándolos en una posición de cierta subjetividad internacional ad hoc (Mariño, 1993, p. 187). De hecho, son múltiples los esfuerzos de Naciones Unidas para tratar de evitar las vulneraciones de derechos humanos por parte de estos poderosos actores internacionales, aunque sin lograr sacar adelante el proyecto de tratado sobre la Responsabilidad de las Empresas Transnacionales y otras empresas comerciales en la esfera de los derechos humanos.

Todos estos procesos han impulsado también el uso del soft law más allá de los tradicionales memorandos de entendimiento -MOU- (los acuerdos internacionales no normativos de la Ley 25/2014). Se trata de aquellas normas que carecen de vinculatoriedad jurídica conforme se entiende en los contenidos clásicos del derecho (Garrido Gómez, 2017, p. 59), porque están debilitadas bien en su obligatoriedad bien en su precisión. Ofrecen diferentes ventajas respecto al hard law, como nos explican Abbott y Snidal (2000, p. 423): con frecuencia son más fáciles de alcanzar (sobre todo en temas en los que los Estados son especialmente celosos de soberanía), ofrecen modos más efectivos para lidiar con la incertidumbre (sobre todo en temas en los que se va a ir aprendiendo a medida que se implementen los acuerdos), y facilitan el compromiso y, por tanto, la cooperación entre actores con diferentes intereses, valores, poder y subjetividad jurídica. En el ámbito de la Unión Europea, las recomendaciones y los dictámenes sirven para coadyuvar al cumplimiento del derecho (además de para ayudar a su interpretación en sede judicial), y múltiples acuerdos de soft law sirven para la cooperación entre las instituciones entre sí y con los Estados, o para llegar acuerdos con terceros que no podrían existir como tratados (Garrido Gómez, 2017, p. 81).Todo ello sin prejuicio de la posibilidad de que acaben evolucionando en normas en sentido estricto, recurribles ante sistemas de solución de controversias. Pues hoy es aceptado que no constituyen 
una fuente nueva de derecho internacional y que de acabar siendo obligatorias para los Estados es porque se han reconducido "a alguna de sus fuentes, sobre todo a los tratados o la costumbre internacional” (Garrido Gómez, 2017, p. 60).

\subsection{La institucionalización y humanización del derecho internacional}

El proceso de institucionalización del derecho internacional es creado por los propios Estados con la proliferación de las organizaciones internacionales, principalmente a partir de la II Guerra mundial. Este es el más importante factor de transformación del derecho internacional, ya que el progreso de las normas imperativas y la consiguiente jerarquización normativa tiene origen en este proceso, en un ordenamiento hasta entonces caracterizado por su horizontalidad (Carrillo Salcedo, 1976, p. 285).

En concreto, fue decisivo el nacimiento de la Organización de las Naciones Unidas y su objetivo fundamental de paz, principalmente a través de la consolidación en su Carta del principio de la prohibición de la amenaza y el uso de la fuerza, y la promoción del desarrollo del derecho internacional de los derechos humanos. De hecho, es una noción clásica continuamente renovada (Carrillo Salcedo, 1976, p. 48) la existencia de un vínculo estrecho e indiscutible entre el respeto de los derechos humanos en el interior del Estado y el mantenimiento de la paz entre los Estados.

La institucionalización supone: el paso de un derecho internacional con el Estado como protagonista único, al reconocimiento de la personalidad jurídica internacional de las organizaciones internacionales y la apertura de la reflexión sobre la personalidad de otros actores en un debate que continúa abierto; la convivencia del bilateralismo con el multilateralismo; el paso de la concepción del ordenamiento con la finalidad de permitir la coexistencia entre sujetos, al fomento de la cooperación entre los mismos; y, por tanto, la transición de normas de delimitación competencial indiferentes a las diferencias ideológicas, a la demanda de cierta homogeneidad de valores. Como explica sintéticamente el profesor Alcaide Fernández (2005, p. 91), la presencia e intensificación de esos valores e intereses comunes, promovidos por estas organizaciones internacionales, se traduce en el derecho internaional contemporáneo en el distinto rango jerárquico de las normas (normas dispositivas y normas imperativas o de ius cogens), y en la distinta naturaleza y alcance de las obligaciones (obligaciones con efectos bilaterales y las obligaciones erga omnes, es decir, las obligaciones de los Estados hacia la comunidad internacional en su conjunto).

La jerarquía de las normas en el derecho internacional contemporáneo se expresa en el artículo 103 de la Carta de las Naciones Unidas y, sobre todo, en el reconocimiento de normas imperativas en los artículos 53 y 64 de la Convención de Viena de 1969 sobre el Derecho de los Tratados, declarando nulos los tratados contrarios a las mismas, sin importar el íter temporal. Se trata de normas, que como expresión de intereses y valores supremos de la comunidad internacional, se imponen al resto de normas del ordenamiento jurídico condicionando su validez. Parece existir consenso en considerar, al menos, normas de esta naturaleza, las referidas al mantenimiento de la paz y la seguridad internacionales; a la existencia y a la igualdad de los Estados; el derecho de libre determinación de los pueblos, y el núcleo básico de los derechos humanos (Casado Raigón, 1999, p. 64). Se trata de normas de derecho internacional general y oponibles erga omnes, de modo que la exigencia de la responsabilidad por su violación deviene universal, más allá de las víctimas concretas. Aunque no se pretenda una correlación exacta entre las normas de ius cogens y las responsabilidades erga omnes (Alcaide Fernández, 2005, p. 95), no es difícil aceptar que de las normas imperativas dimanan obligaciones oponibles a todos 
(Casado Raigón, 1999, p. 57). Sin embargo, no todo el derecho internacional general está compuesto por normas imperativas. Dentro de este, destacan los principios estructurales y fundamentales del derecho internacional, que, lejos de ser principios generales del Derecho, son normas básicas de este ordenamiento jurídico (Jiménez Piernas, 2011, p. 61). Recogidos originariamente en el artículo 2 de la Carta de las Naciones Unidas, hoy tienen su expresión formal más completa en la Resolución 2625 (XXV) de la Asamblea General de Naciones Unidas, aunque ésta no tiene carácter exhaustivo. No es casualidad la coincidencia entre las normas imperativas del derecho internacional y algunos de estos principios estructurales.

No son tampoco desdeñables los efectos de la institucionalización del derecho internacional en los mismos procesos de creación normativa. En primer lugar, destaca la labor de codificación de la costumbre internacional. Aunque se trata de una actividad con precedentes públicos y privados (Irigoin, 2005, p. 181), cobra una dimensión totalmente distinta cuando la Carta de las Naciones Unidas (artículo 13.1.a) establece que la Asamblea General promoverá estudios y hará recomendaciones para, entre otros, impulsar el desarrollo progresivo del derecho internacional y su codificación, labor que ha venido realizando la Comisión de Derecho Internacional de Naciones Unidas. El tratado también ha cobrado protagonismo por ser el instrumento principal a través del cual se ha procedido a la codificación, clarificando y precisando el derecho y, por tanto, aumentando la seguridad jurídica (Díaz Barrado, 1995, pp. 35, 36 y 46$)$.

También es relevante, en segundo lugar, el surgimiento de un nuevo tipo de norma internacional consuetudinaria. Las resoluciones en principio no jurídicamente vinculantes de la Asamblea General de Naciones Unidas o de conferencias internacionales generalmente impulsadas por esta organización, con frecuencia sirven de expresión del elemento espiritual u opinio iuris de estas normas jurídicas (Pastor Ridruejo, 2019, p. 76). De modo que, tras producirse la consiguiente adecuación del comportamiento de los Estados a esas expresiones de voluntad, nace una costumbre en un período de tiempo mucho más corto que en el derecho internacional clásico, en un proceso en el que el elemento espiritual ha precedido al elemento material (también de manera novedosa) y contando con un documento escrito de referencia que les permite una precisión mucho mayor. Hay que tener en cuenta que este proceso, junto al de la codificación y la proliferación de tratados multilaterales, ha llevado a que determinadas normas consuetudinarias sean coincidentes con normas recogidas en normas de carácter convencional (ora anteriores, como desencadenantes, ora posteriores a la norma consuetudinaria). Ello no impide que ambas gocen de autonomía y obliguen a quien corresponda según su propia naturaleza.

En tercer lugar, hay que tener en cuenta la capacidad de las organizaciones internacionales para crear derecho, lo que en el caso de las Naciones Unidas y sus organismos especializados tiende a tener un alcance universal. Sin embargo, pocas veces las organizaciones internacionales tienen competencias para poder crear Derecho obligatorio para sus Estados miembros, e incluso directamente aplicable a los particulares bajo su jurisdicción, como sí sucede y de manera profusa en el caso de la Unión Europea. En el ámbito de Naciones Unidas, hay que destacar el papel que puede jugar el Consejo de Seguridad. De acuerdo a la Carta de las Naciones Unidas (artículos 24 y 25), este órgano puede, a fin de a mantener la paz y la seguridad internacionales, adoptar decisiones que los miembros de Naciones Unidas aceptan cumplir. En un principio, se entendió que se trataba, principalmente, de medidas sancionadores dirigidas a Estados concretos, primero, y a organizaciones privadas o personas, después. Con la Resolución 1373 (2001), de 28 de septiembre (reacción a los ataques terroristas a Estados Unidos el 11 de septiembre de 2001), el 
Consejo de Seguridad estableció normas para combatir el terrorismo, jurídicamente vinculantes para todos los Estados (en un contexto totalmente distinto a las medidas adoptadas hasta ese momento para disciplinar a un Estado concreto), convirtiéndose en una suerte de legislador de la comunidad internacional. La Resolución 1540 (2004) confirmó esta posibilidad, que, en la medida en que supone la creación de derecho internacional general más allá de las categorías clásicas de la costumbre y el tratado, ha sido calificado de revolucionaria (Lavalle, 2004, p. 436).

Por último, no podemos ignorar el papel de la amplia jurisprudencia de los numerosos tribunales internacionales en la determinación y precisión (incluso la creación) de la norma internacional (Von Bogdandy y Venzke, 2012, pp. 18-19; Zarviyev, 2012, p. 7). Aunque el referente habitual de las fuentes del derecho internacional, el artículo 38 del Estatuto del Tribunal Internacional de Justicia, considera la jurisprudencia como mero medio auxiliar para la determinación de las reglas de Derecho, y se niegue otro valor que su obligatoriedad para las partes en litigio en el caso concreto (artículo 59 del Estatuto), lo cierto es que el papel de estos órganos ha ido mucho más allá, adoptando un papel proactivo. El propio Tribunal Internacional de Justicia ha jugado un papel fundamental en la confirmación de la existencia de instituciones fundamentales como las normas de ius cogens y la responsabilidad erga omnes. Su relevancia tiene origen en las propias características del ordenamiento jurídico internacional, en el que los jueces no solo han tenido que interpretar la norma, sino también con mucha frecuencia han de constatar y precisar la existencia de normas consuetudinarias o de los mismos principios generales y los principios estructurales del derecho internacional. Las cortes internacionales son también decisivas en la confirmación del carácter de ius cogens y erga omnes de ciertas normas, y también destaca su papel en el impulso de la transformación del soft law en hard law, la expansión de la relación entre costumbre y tratado, o dotando de autoridad al proceso de codificación de la Comisión de Derecho Internacional incluso antes de la finalización de su trabajo (Boyle y Chinkin, 2007, p. 269).

Por otro lado, el proceso de institucionalización y correlativa humanización del derecho internacional también incide en su fundamento al poner de manifiesto un aspecto objetivo (más allá del subjetivismo protagonizado por los Estados). Sin embargo, podemos considerar que dicho fundamento objetivo siempre existió en la medida en que es imprescindible para la misma existencia de este ordenamiento jurídico. Podemos identificarlo con la Grundnorm kelseniana, que el profesor austriaco primero fijó en el pacta sunt servanda como regla de derecho natural (Kelsen, 1953, p. 131) y, finalmente, una norma "que establezca a la costumbre constituida por el comportamiento recíproco de los Estados como hecho productor de derecho" (Kelsen, 1986/1960, p. 327). Los mismos principios de igualdad soberana de los Estados y de buena fe son fundamentales en el derecho internacional. La particularidad del derecho internacional contemporáneo es que esos elementos objetivos se amplían, y se agrandan así los límites a la voluntad del Estado, y, por tanto, al relativismo del derecho internacional.

En este sentido, es paradigmático el derecho internacional de los derechos humanos, expresión indiscutible de ese proceso de institucionalización y consiguiente humanización del derecho internacional, pues nos permite observar cómo el reconocimiento de los derechos humanos «penetra en el corazón mismo de la soberanía, es decir, en las relaciones de un Estado con las personas que se encuentre bajo su jurisdicción, incluidos sus nacionales, con lo que el rostro de la soberanía queda remodelado y transformado» (Carrillo Salcedo, 1999, p. 20).

Es importante señalar que, en la medida en que la soberanía estatal encuentra algún límite, se trata también de una soberanía más sujeta al derecho. De este modo, 
aunque la soberanía de los Estados sigue siendo un principio estructural del derecho internacional (hay quienes defienden incluso que la norma fundamental), esta se formula, al menos en el derecho internacional contemporáneo, como libertad del Estado limitada por el derecho internacional nacido del propio consentimiento de los Estados. «Esto es así porque la igualdad soberana se configura como una igualdad ante el Derecho más que como una igualdad de derechos» (Espósito, 2009, p. 297).

\section{El Derecho Internacional Público como sistema}

Actualmente, resulta relevante para precisar el concepto de norma internacional la concepción de sistema del derecho internacional (entendido como conjunto interrelacionado en lugar de una mera adición de normas) y su vinculación al problema de la fragmentación de este ordenamiento jurídico. En principio, la creación de regímenes internacionales específicos o especiales, la intensificación del regionalismo jurídico y la creación de un buen número de órganos jurisdiccionales internacionales como manifestación del pluralismo de la comunidad y el derecho internacional, no impiden mantener la unidad del ordenamiento jurídico internacional, debido a la existencia de normas primarias universales (en cuyo núcleo básico estarían las normas relativas a los derechos humanos fundamentales) compatibles con la autonomía relativa de los regímenes especiales. De este modo, el peligro de fragmentación solo existiría ante la aplicación del derecho sin conocimientos suficientes (Rodrigo y García, 2011, pp. 19, 23).

En especial, se ha escrito mucho sobre las posibles amenazas a la unidad del ordenamiento jurídico internacional por la proliferación actual de tribunales internacionales. Un estudio realizado por Charney $(1998$, p. 347) analizando la labor de múltiples tribunales, concluyó que, aunque el incremento del número de cortes internacionales sin un sistema jerárquico efectivo entre ellas hace imposible una uniformidad completa de las decisiones, «estos tribunales están claramente comprometidos con la misma dialéctica. Los fundamentos del derecho internacional general permanecen iguales sin importar cuál es el tribunal que decide el caso».

Sin nos centramos de nuevo en el derecho internacional de los derechos humanos, pese a la primera apariencia, observamos que no solo no se produce fragmentación, sino que parece ir configurándose una interrelación entre todos los niveles jurídicos, internacionales y domésticos, dedicados a la protección de la dignidad de la persona. Esto podría constituir un primer paso en la evolución hacia un derecho global de los derechos humanos, en la medida en que los convenios y los tribunales regionales están creando una misma cultura jurídica a partir de la generación de estándares comunes (García Roca y Carmona Cuenca, 2017, p. 35). Los tribunales internacionales de derechos humanos no solo tienen en cuenta los acuerdos e instrumentos formalmente relacionados con él, sino también el sistema dentro del cual se inscriben, es decir, el conformado por todos los instrumentos en la materia, las decisiones de comités y tribunales que trabajan con ellos, y el derecho internacional en su conjunto.

Podemos encontrar un diálogo judicial en sentido más estricto (Bustos Gisbert, 2019, p. 48) en el ámbito europeo, pues, en la relación entre el Tribunal Europeo de Derechos Humanos, el Tribunal de Justicia de la Unión Europea y los tribunales constitucionales de los Estados miembros de la Unión, no solo se produce una asunción del derecho internacional universal (consuetudinario, convencional, resoluciones del Consejo de seguridad...), sino que, además, se trata de conflictos en los que confluyen varios ordenamientos jurídicos con sus propios órganos judiciales supremos. Así, la decisión que se adopte en cualquiera de ellos tiene 
efectos en los otros ordenamientos jurídicos. Algo similar sucede con la Corte IDH y algunos tribunales constitucionales como el colombiano, en un diálogo judicial transformador y promotor del respeto de los derechos humanos que ha llevado a hablar de un lus Constitutionale Commune (von Bogdandy, 2015).

Ahora bien, los adjetivos internacional y global por definición no pueden conceptualmente estar referidos solo a Europa y América. ¿Podemos ampliar estas reflexiones al resto de los Estados del mundo, a la sociedad internacional en su conjunto, base social del derecho internacional? Anthea Roberts (2017), en su libro Is International Law International? muestra el resultado de entrevistar a iusinternacionalistas en ejercicio y académicos de los cinco Estados miembros permanentes del Consejo de Seguridad de Naciones Unidas, para constatar que el derecho internacional no puede considerarse realmente uno y universal, en la medida en que es entendido, interpretado, aplicado y explorado de formas muy distintas por actores de diferentes Estados. En las instituciones universales es evidente el predominio de occidente y del inglés como lengua vehicular, pero eso no impide que Estados tan relevantes como Rusia y China muestren discrepancias sobre cuestiones tan importantes como que la soberanía estatal no ha de ceder ante los derechos humanos, por seguir en este ámbito del derecho internacional. Esta realidad nos recuerda la importancia de la política en el derecho internacional, y de dónde pueden provenir las verdaderas amenazas a la unidad del ordenamiento jurídico internacional.

\section{Reflexiones finales}

La norma internacional ha experimentado múltiples cambios en los últimos setenta años. La costumbre y el tratado se han transformado, ha cobrado protagonismo el derecho de las organizaciones internacionales y ha proliferado un nuevo tipo de regla, el derecho blando o soft law, que con independencia de su evolución o no hacia una fuente cierta de derecho como la costumbre o el tratado, también juega una función en la regulación de las relaciones internacionales. Los tribunales internacionales, múltiples y activos, juegan un papel determinante en la precisión y determinación de la existencia de la norma internacional y en su caracterización, sin que su pluralidad y falta de jerarquización haya deshecho la unidad sistémica del ordenamiento jurídico.

Desde el punto de vista del contenido material, la norma internacional también se ha enriquecido en un doble sentido. Por un lado, ha ampliado el ámbito regulatorio en respuesta a los desafíos planteados por un mundo cada más interdependiente y, por tanto, necesitado de regulación internacional, especialmente para la cooperación. De ahí, la dificultad de encontrar ámbitos sustantivos reservados a la regulación intraestatal. Por otro lado, la norma internacional se ha nutrido de valores. No solo juega un papel de regulación de relaciones entre Estados, sino que impone la protección de intereses propios de toda la comunidad internacional sobre el sustrato de unos valores comunes básicos.

Estos cambios sufridos por la norma internacional están relacionados también con el incremento de las voces que se expresan a través de este ordenamiento jurídico. La globalización permite que actores más o menos poderosos jueguen cierto papel en el proceso de creación normativa. Las organizaciones internacionales, nuevos sujetos de derecho internacional, lideran el proceso de transformación de la creación de las normas y modificación del contenido de las mismas, previamente referidos, al constituirse en los marcos propicios de defensa de intereses colectivos y comunitarios: defensa de la paz y la seguridad internacionales, los derechos humanos, el derecho al desarrollo, la protección del medio ambiente, etc. 
De este modo, aunque en última instancia siempre encontremos al Estado, nacen normas que suponen un límite a su soberanía. Más aún si se trata de normas imperativas o principios estructurales del derecho internacional. De este modo, la soberanía estatal encuentra en la norma internacional, de manera paralela a los límites fácticos de la globalización, un límite jurídico más allá de las obligaciones relacionales asumidas directamente por el Estado.

En conclusión, la norma internacional contemporánea tiene una caracterización rica y compleja, donde confluyen formas antiguas y nuevas, contenidos y funciones clásicos e innovadores.

\section{Bibliografía}

Abbott, Kenneth W. y Snidal, D. (2000). Hard and Soft Law in International Governance. International Organization, 54 (3), pp. 421-456.

Alcaide Fernández, J. (2005). Orden público y Derecho Internacional: desarrollo normativo y déficit institucional. En Salinas de Frías, A. y Vargas Gómoz-Urrutia, M. (Ed.), Soberanía del Estado y Derecho internacional. Homenaje al profesor Juan Antonio Carrillo Salcedo. Tomo I (pp. 91-115). Sevilla, España: Servicios de publicaciones de las Universidades de Sevilla, Córdoba y Málaga.

Boyle, A. y Chinquin, C. (2007). The Making of International Law. Nueva York, Estados Unidos: Oxford University Press.

Bustos Gisbert, R. (2019). La noción de diálogo judicial en el espacio judicial europeo. En Martín y Pérez de Nanclares, J. (dir.) y González Herrera, D. (coord.), El diálogo judicial internacional en la protección de los derechos fundamentales (pp. 45-68). Valencia, España: Tirant lo Blanch.

Carrillo Salcedo, J. A. (1999). Dignidad frente a barbarie. La Declaración Universal de Derechos Humanos cincuenta años después. Madrid, España: Trotta.

Carrillo Salcedo, J. (1997). Reflections on the Exitence of a Hierarchy of Norms in International Law. EJIL, Vol. 8 (4), pp. 583-595.

Carrillo Salcedo, J. (1976). Soberanía del Estado y Derecho Internacional. Madrid, España: Tecnos.

Casado Raigón, R. (1999): Notas sobre el ius cogens internacional. Córdoba, España: Servicio de Publicaciones de la Universidad de Córdoba.

Charney, J. (1998). Is Internatinal Law Threatened by Multiple International Tribunals? Recueil des cours, Vol. 271, pp. 101-382.

Cotterrell, R. (2012). What is transnational law? Law \& Social Inquiry, Vol. 37 (2), pp. 500-524.

Del Arenal, C. (2009). Mundialización, creciente interdependencia y globalización. Cursos de Derecho internacional y Relaciones internacionales de Vitoria Gasteiz 2008 (pp. 181-268). Bilbao, España: Servicio editorial de la Universidad del País Vasco.

Díaz Barrado, Castor M. (1995). La necesidad de la codificación en el Derecho internacional público. REDI. Vol XVII (I), pp. 33-53.

Díez de Velasco, M. (2017). Instituciones de Derecho Internacional Público. Madrid, España: Tecnos.

Espósito Massicci, C. D. (2009). Soberanía e igualdad en el derecho internacional. Anuario de la Facultad de Derecho de la Universidad Autónoma de Madrid (13), pp. 291-310.

García Roca, J. y Carmona Cuenca, E. (eds.) (2017). ¿Hacia una globalización de los derechos? El impacto de las sentencias del Tribunal Europeo y de la Corte Interamericana. Pamplona, España: Thomson Reuters Aranzadi. 
Garrido Gómez, Ma I. (2017). El soft law como fuente del Derecho extranacional. Madrid, España: Dykinson.

Irigoin Barrenne, J. (2005). La codificación de la costumbre internacional. Revista De La Facultad De Derecho (8), pp. 181-194.

Kelsen, H. (1986/1960). Teoría pura del Derecho. México D. F., México: UNAM, Instituto de Investigaciones Jurídicas.

Kelsen, H. (1953). Théorie du droit international public. Recueil des Cours, Vol. 84 (III), pp. 1-201.

Kelsen, H. (2013/1952). Principios de Derecho Internacional Público. Granada, España: Comares.

Koh, Harold Hongju (1996). Trasnational Legal Process. Faculty Scholarship Series. Paper 2096, pp. 181-207. Recuperado de https://digitalcommons.law.yale.edu/fss_papers/2096.

Lavalle, R. (2004). A Novel, If Awkward, Exercise in International Law-Making: Security Council Resolution 1540 (2004). Netherlands International Law Review, 51 (3), pp. 411-437. doi:10.1017/S0165070X04004115

Mariño Menéndez, F. M. (1993). Derecho internacional público. Madrid, España: Trotta.

Müller, T. (2008). Customary Transnational Law: Attacking the Last Resort of State Sovereignty. Indiana Journal of Global Legal Studies, Vol. 15 (1), pp. 19-47.

Palomares Lerma, G. (2006): Relaciones internacionales en el siglo XXI. Madrid, España: Tecnos.

Pastro Ridruejo, J. A. (2019). Curso de Derecho internacional público y organizaciones internacionales. Madrid, España: Tecnos.

Roberts, A. (2017). Is International Law International? Nueva York, Estados Unidos: Oxford University Press.

Rodrigo, A. J. y García, C. (2011). La vuelta a la teoría por medio del diálogo científico. En Rodrigo, A. J. y García, C. (eds.), Unidad y pluralismo en el Derecho Internacional Público y en la Comunidad Internacional. Coloquio en homenaje a Oriol Casanovas. Barcelona, 21-22 de mayo de 2009 (pp. 15-40). Madrid, España: Tecnos.

Von Bogdandy, A. (2015). lus constitutionales commune en América Latina: Una mirada a un constitucionalismo transformador. Derecho del Estado (34), pp. 3-50. doi: 10.18601/01229893.n34.01.

Von Bogdandy, A. y Venzke, I. (2012). In whose name? An investigation of International Court's public authority and its democratic justification. The European Journal of International Law, 23 (1), pp. 7-41. doi: 10.1093/ejil/chr106

Zarbiyev, F. (2012). Judicial activism in international law: A conceptual framework for analysis. Journal of International Dispute Settlement, 3 (2): 1-32. doi: 10.1093/jnlids/ids005. 